\title{
Zur Geschichte der Staublunge
}

Nach zwei Vorträgen an der Universität Zürich, Juni 1974

Von Erich Posner

Zweifellos sind die Mineralstaublungen die älteste Berufskrankheit der Menschheit. Der paläolithische Mensch sammelte Kieselsteine an den Ufern von Flüssen und Meeren zur Herstellung seiner Waffen und Werkzeuge. Der neolithische Mensch entdeckte, daß die noch wasserhaltigen Kiesel unter der Erdoberfläche viel geschmeidiger waren; er grub nach ihnen und verarbeitete sie in seinen kalten Höhlen. Da besteht wohl kein Zweifel, daß sich in seinen Lungen silikotische Aggregate bildeten. Natürlich wissen wir nichts über die Silikosesterblichkeit der prähistorischen Menschen; aber Collis hat 1915 nachgewiesen, daß eine Gruppe der linearen Nachfolger unserer Ahnen in der englischen Grafschaft Suffolk, die sogenannten «flint-knapper», verglichen mit der allgemeinen Bevölkerung eine siebenfache Sterblichkeit an Brustkrankheiten aufwiesen (Tabelle 1). Als dann endlich im ersten Jahrzehnt unseres Jahrhunderts die Brustkrankheiten der Flintknapper als Silikose und Siliko-Tuberkulose erkannt wurden, beschäftigte die Industrie nur noch ein paar alte Männer.

Tabelle 1. Mortalität der Flintknapper aus Brandon (Suffolk), verglichen mit anderen Berufen (nach Collis, 1915). Alle Todesursachen zusammen $=100 \%$

\begin{tabular}{lclc}
\hline & Phthisis & $\begin{array}{l}\text { Andere } \\
\text { Brustkrankheiten }\end{array}$ & $\begin{array}{l}\text { Andere } \\
\text { Gründe }\end{array}$ \\
\hline $\begin{array}{l}\text { Flintknapper } \\
\begin{array}{l}\text { Frauen und Witwen } \\
\text { der Flintknapper }\end{array}\end{array}$ & 77,8 & 7,4 & 14,8 \\
$\begin{array}{l}\text { Brandon-Distrikt } \\
\text { Alle Männer aus England } \\
\text { und Wales, 1900-1902 }\end{array}$ & 0,0 & 15,4 & 84,6 \\
\hline
\end{tabular}

Die Identifizierung industrieller Krankheiten erfolgte oft sehr langsam. Zum Beispiel war es seit der Eroberung Afrikas durch europäische Mächte bekannt, daß viele Frauen der Bantus in Ostafrika von chronischen und oft tödlichen 
Lungenkrankheiten betroffen waren, während die Männer selten solchen Leiden unterlagen. Erst 1967 haben Palmer und Daynes festgestellt, daß diese Mädehen und Frauen an schweren Silikosen litten, die sie beim Zermahlen von Korn und Mais zwischen Quarzsteinen in schlecht gelüfteten Hütten erworben hatten.

\section{Altertum}

Bis zur Zeit der ersten industriellen Revolution im 18. Jahrhundert hören wir von beruflichen Brustkrankheiten beinahe ausschließlich mit Bezug auf den Bergbau. Vor etwa sechstausend Jahren begannen die Ägypter ihre Expeditionen nach der Halbinsel Sinai und nach Nubien auf der Suche nach Gold, Silber, Kupfer und anderen wertvollen Mineralien. Diese Erze wurden aus Quarz und Sand ausgegraben, und diese bestehen fast ganz aus freier Kieselsäure.

Aus dieser Zeit gibt es wenig medizinische Berichte über die Krankheiten der Häuer; aber der griechische Historiker Diodorus Siculus schrieb im ersten Jahrhundert vor Christus: «Die Pharaonen schickten in die Gruben Verbrecher, Kriegsgefangene, Irrsinnige und die niedrigsten Sklaven. An den Füßen gefesselt, mußten sie Tag und Nacht in den Gruben bleiben. Junge Burschen holten unter großen Anstrengungen die Steinbrocken an die Oberfläche» (nach Rosen 1943).

Zweitausend Jahre später finden wir in einem parlamentarischen Bericht einer englischen Untersuchungskommission eine Zeichnung (Abb.1), die mit dem obigen Zitat völlig vereinbar ist (Hansard).

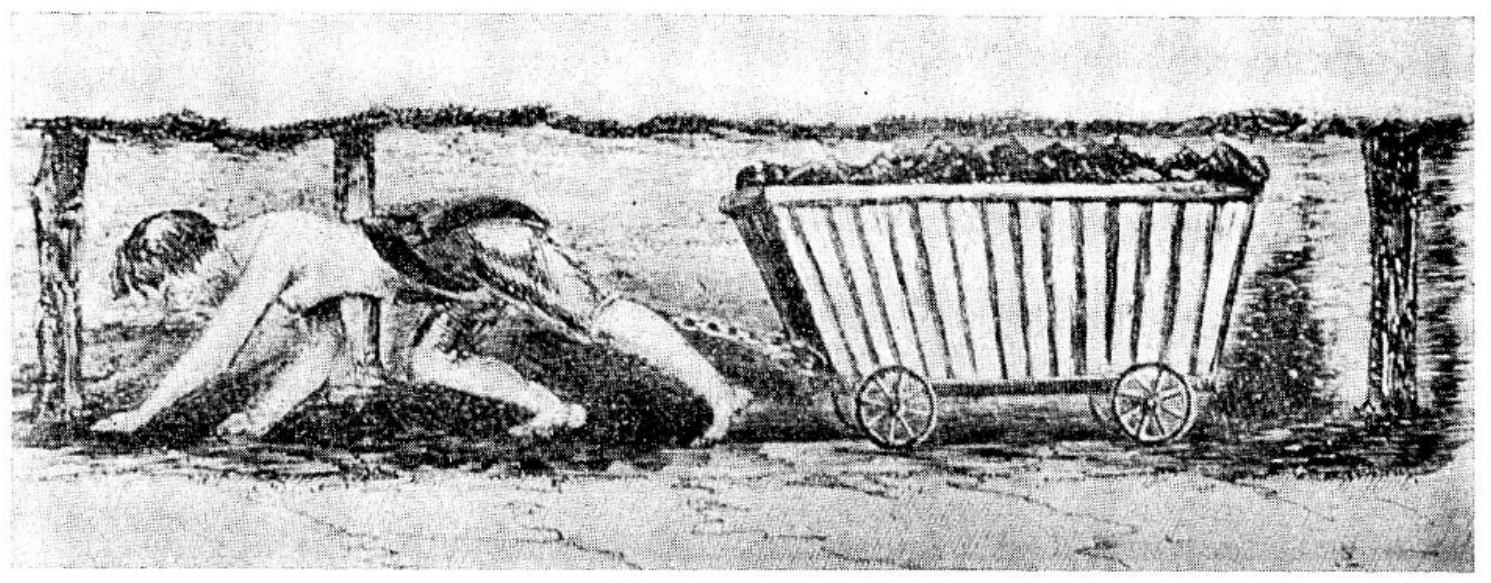

Abb. 1. In einer schottischen Grube des 19. Jahrhunderts 
Griechische Zivilisation und römischer Imperialismus verbreiteten die Grubenarbeit in Europa und Asien, besonders in Karthago, Iberien, an der adriatischen Küste, in Frankreich und England.

Der römische Naturforscher und Dichter Titus Lucretius Carus stellte in seinem Lehrgedicht «De rerum natura» (um 55 v.Chr.) die Frage: «Siehst du und hörst du nicht, in welch kurzer Zeit sie [die Bergarbeiter] zugrunde gehen und wie ihre Arbeit sie bleicht?» Der Konsul Silius Italicus schrieb im ersten Jahrhundert n. Chr.: «Das Angesicht des asturischen Häuers ist so blaß wie das Gold, das er der Erde entreißt.» Plinius (23-79 n.Chr.) erwähnt in seiner Naturgeschichte, daß sich die Grubenarbeiter gegen die Staubeinatmung mit Tierblasen schützen. Kaiser Justinian faßte um 530 zusammen: «In die Gruben geschickt zu werden, bedeutet ein Todesurteil» (nach Rosen).

\section{5. bis 17. Jahrhundert}

Der Zusammenbruch des Römischen Reiches verursachte den Verfall des europäischen und asiatischen Bergbaus. Ulrich Ellenbogen (gest. 1499) hinterließ ein Buch «Von den gifftigen, besen Tempffen und Reuchen», das erst 25 Jahre nach seinem Tode veröffentlicht wurde, dann aber viele Nachdrucke erlebte. Ellenbogen stand noch fest auf galenischem Boden und gab den Bergleuten den wahrscheinlich überflüssigen Rat, viel zu schwitzen und viel Wein zu trinken. Über die Therapie jener Zeit verdanke ich wertvolle Angaben Herrn Dr. Schiller aus Frankfurt. Meistens wurden den Häuern Expectorantia und Zimt, Ziegenmilch, Fichtennadeln, Nußkerne und der auch uns bekannte Eibischtee verschrieben.

Vom 11. bis zum 14. Jahrhundert fand man reiche Erzflöze im Erzgebirge, das Böhmen von Sachsen trennte. Auf der böhmischen Seite entwickelte sich Joachimsthal, auf der sächsischen Annaberg zu einem wichtigen Zentrum des Bergbaus. Kaiser, König, Pfalzgraf und Condottiere brauchten enorme Summen von Silberund Goldmünzen, und der Erzgebirger Bergbau gewann große Bedeutung.

Einige Stadtphysici im Erzgebirge beschäftigten sich mit den Krankheiten der Bergarbeiter, vor allem Georg Bauer, genannt Agricola (1494-1555). Er war ein Renaissancemensch par excellence, Arzt, Politiker, Technologe, Künstler und Philosoph. Im 12. Buch seines monumentalen Werkes «De re metallica» (1556) berichtete Agricola über die zunehmende Tiefe und Trockenheit des böhmischsächsischen Bergbaus und fügte bei: «Der Staub dringt in die Luftwege und bewirkt schweres Atmen, sogar Lungengeschwüre und Schwindsucht.» Und an einer anderen Stelle: «Beispielsweise wurden bei den Bergleuten Frauen gefun- 
den, die siebenmal verheiratet waren. So rasch waren ihre Männer an Lungenkrankheiten gestorben.» Es ist klar, daß Agricola hier von Silikose und SilikoTuberkulose spricht; aber er konnte nicht wissen, daß viele Erzgebirgsgruben mit Radongas verseucht waren. Viel später fand man, daß viele Bergleute im Erzgebirge an Lungenkrebs starben (Harting und Hesse 1879, Löwy 1929).

Noch Jahrhunderte später wurde Agricolas Werk von vielen Naturforschern, z. B. von Goethe, bewundert. Ein zukünftiger Präsident der Vereinigten Staaten, Herbert Hoover, hat es ausgezeichnet ins Englische übersetzt. Agricolas Leitmotiv : «Es ist notwendig, daß man alletwegen mehr auf die Gesundheit als auf den Gewinn achte », ist leider bis in unsere Zeit oft ein frommer Wunsch geblieben.

Das stürmische Leben des Theophrastus Bombastus von Hohenheim, genannt Paracelsus (1493-1541), ist wohlbekannt. Er stand gleichsam zwischen den langsam austrocknenden Flüssen des Mittelalters und den frischen reißenden Strömen der Renaissance. Er verbrannte die Schriften von Galen und Avicenna vor der Basler Kathedrale und forderte, daß die Alchimisten Heilmittel und nicht Gold herstellen sollten. Während seiner langen Wanderungen hielt sich Paracelsus in Grubengebieten auf, und von dort stammt sein Buch «Von der Bergsucht oder Bergkranckheiten drey Bücher». Es wurde ebenso wie das Werk Agricolas erst nach seinem Tode publiziert (1567). Dieser mächtige Bilderstürmer hatte jedoch die Ketten der Altertumsmedizin noch nicht gesprengt. Er erkannte wohl, daß die Bergsucht eine spezifische Krankheit der Bergarbeiter war, die nach seiner Ansicht auf dem «Nebel im Berg» oder dem «Chaos» beruhte. Sehr stark rührte sich in ihm der Mensch des Mittelalters, wenn er behauptete, daß dieses Chaos von astralen Konstellationen abhinge und die Symptome der Bergkrankheit auf der Sedimentation von Schwefel, Salz und Quecksilber, dem sogenannten Tartarus, beruhten. Man soll den Tartarus aus den Lungen durch häufiges schweres Schwitzen herauswaschen. An dieser Stelle ist eines seiner Rezepte zur Verhütung der Bergsucht interessant (Der viert tractat, Das drit capitel): «alle monat ein mal ...

Liquoris tartari unz. ij.

Olei colcotarini scrup. j.

Laudani purissimi drach. semis.

gemischt und eingeben dreier gerstenkorn schwer». Wir müssen aufrichtig eingestehen, daß die klinische Pharmakologie der folgenden 400 Jahre keine besseren Verhütungsmittel der Silikose zur Verfügung gestellt hat.

Die großen Schriften Agricolas und des Paracelsus überschatteten eine ganze Reihe kleinerer Werke. Ich kann hier nur von wenigen berichten.

Martin Pansa wirkte im 17. Jahrhundert in Annaberg, und sein «Consilium 
peripneumoniacum» (1614) enthält lange Plagiate aus dem Werk von Paracelsus. Er beschrieb aber recht gut die Symptome der Bergkrankheit: Dyspnoe, Husten, Melanoptysis und Brustdruck. Wie so viele seiner Zeitgenossen war Pansa ein eifriger Moralist: «Eye so schmeißt nur, liebe Bergleute, mit Freuden den Schweiß zu bewegen, nicht aber untereinander in den Bierhäusern, auch nicht auf Euere Weiber, denn ihr habt genug zu schmeißen, das feste Gestein.»

Die erste recht genaue Beschreibung einer silikotischen Lunge verdanken wir dem Holländer Ysbrand van Diemerbroeck (1609-1674), der 1672 schrieb : «Als ich das Skalpell durch eine Steinmetzlunge zog, schien es mir, als ob ich durch einen Sandkörper schnitte». Diemerbroeck war meines Wissens auch der erste, der auf die Bedeutung der quantitativen Staubmessung hinwies: «... ein Steinmetz hängte in seiner Werkstatt eine Rinderblase auf, in welcher er nach einem Jahre eine Handvoll Staub fand, die einen unvorsichtigen Arbeiter töten konnte.»

Der Vater der Arbeitsmedizin, Bernardino Ramazzini (1633-1714), zitierte in seinem Buch «De morbis artificum» die bedeutendsten Beiträge seiner Zeitgenossen und nahm eine recht fatalistische und pessimistische Stellung ein: «Die Bergleute verfallen gräßlichen Krankheiten, die sich aller Kur widersetzen. Ja, man muß sich wirklich fragen, ob es gut und nach Gottes Willen ist, diesen armen Kreaturen Medikamente zu verschreiben und so ihr elendes Leben zu verlängern.» Jedoch in einem weiteren Kapitel fühlen wir, daß die industrielle Revolution und der Frühkapitalismus schon laut an die Tore der alten Welt klopften: «... Jedoch da Kaufleute und Prinzen von den Gruben großen Nutzen ziehen und Metalle für die meisten Handwerke nötig sind, müssen wir - wenn es möglich ist - das Leben der Bergleute verlängern.»

\section{Die neuere Porzellan-, Asbest- und Kohlenindustrie}

Wenn ich die neuen Gefahren der industriellen Revolution schildern will, muß ich mich an dieser Stelle auf die Porzellanindustrie, die Asbestgefahr und auf die Brustkrankheiten der Kohlenarbeiter beschränken.

Für viele Ärzte war die englische keramische Industrie ein ausgezeichnetes Feld für umfassende Untersuchungen, da etwa $80 \%$ der gesamten Industrie in Stokeon-Trent und seiner Umgebung konzentriert sind und weil die Industrie etwa 30000 Frauen beschäftigt. Bis zum 18. Jahrhundert verfertigten die Töpfer dieser Gegend Gefäße aus einem schweren Ton, der nur wenig freie Kieselsäure enthielt.

Es wird überliefert, daß 1728 ein gewisser Astbury das Danaergeschenk der feingemahlenen Kieselsteine nach Stoke brachte und das Kieselpulver in her- 
metisch verschlossenen Kellern mit Kaolin und Feldspat vermischte (Meiklejohn 1969). Vom ökonomischen und ästhetischen Standpunkt aus war die Einführung des «Flints» ein großer Erfolg, vom Standpunkt des Gesundheitswesens aus eine schwere Katastrophe. Schon sechs Jahre nach Astburys Entdeckung legte ein ortsansäßiger Ingenieur, Thomas Benson, ein Patentgesuch für bewässerte Kieselmühlen vor, das er unter anderem mit den Worten begründete: «Jeder gesunde Mann und Bursche, der das Kieselmahlen im Trockenen betreibt, kann nicht länger als zwei Jahre leben ... So wirkt der tödliche Staub, den er ständig einatmet» (Meiklejohn 1969).

Tabelle 2. Bestandteile englischer keramischer Waren

\begin{tabular}{llllll}
\hline $\begin{array}{l}\text { Irdenes Geschirr, Kacheln } \\
\text { und Toiletteneinrichtungen }\end{array}$ & Porzellan & & «Bone-China " \\
\hline Reines Kaolin & $22-28 \%$ & Reines Kaolin & $20-28 \%$ & Gemahlene \\
Unreines Kaolin & $22-28 \%$ & Unreines Kaolin & $20-28 \%$ & Knochen & $45-52 \%$ \\
Kieselstaub & $32-40 \%$ & Kiesel oder Quarz & $32-35 \%$ & Kaolin & $17-26 \%$ \\
Feldspat & $13-18 \%$ & Feldspat oder & & Feldspat & $24-32 \%$ \\
& & Nephelinsyenit & $18-24 \%$ & &
\end{tabular}

Das feuchte Mahlen des Rohmaterials in der keramischen Industrie setzte sich langsam durch; aber aus Tabelle 2 ist ersichtlich, daß bis zum heutigen Tage die freie Kieselsäure ein hochprozentiger Bestandteil des irdenen Geschirrs, der Badezimmer- und Ofenkacheln und der Toiletteneinrichtungen geblieben ist. Zwar enthält das bekannte englische «Bone-China» nur wenig freie Kieselsäure; aber bis vor 30 Jahren wurde feingemahlener Kieselstaub als Unterstützungsmaterial während des Ofenbrennens verwendet. Die Männer, die die Porzellanwaren vor dem Brennen in den gemahlenen Kiesel einbetteten, und die Frauen, die nach dem Brennen den Kristobalit und Trydimit von den Gefäßen abputzten, waren hoher Silikosegefahr ausgesetzt. Vor etwa 30 Jahren wurde der Kieselstaub durch Aluminat ersetzt, und die Silikosegefahr in diesem Prozeß ist völlig verschwunden (Meiklejohn 1963, Meiklejohn und Posner 1957, Posner und Kennedy 1967). Jedoch im 19. Jahrhundert arbeiteten noch über tausend Kinder lange Stunden in der keramischen Industrie, und Farr stieß 1877 den Hilferuf aus: «Was können wir tun, um die Menschenleben zu retten, die so frühzeitig und schnell bei der Herstellung irdenen Geschirrs zugrunde gehen?» 
Die enorme Verbreitung der Silikose und Mischstaublunge wurde erst im 20. Jahrhundert durch röntgenologische Serienuntersuchungen festgestellt. Viel früher publizierte ein Internist in Stoke, John T.Arlidge (1822-1899), der nie eine Röntgenaufnahme gesehen hat, einen ausgezeichneten, aber von seinen Zeitgenossen bald vergessenen Bericht über Mortalität, Differentialdiagnose und Sektionsbefunde bei keramischen Arbeitern (Arlidge 1892). Dieses Meisterwerk, "The Hygiene, Diseases and Mortality of Occupations", hat keine weiteren Auflagen erlebt.

Die Arbeitsbedingungen in der englischen keramischen Industrie verbesserten sich nur sehr langsam und sind im einzelnen im Bericht über die "Health Conditions in the Ceramic Industry" anläßlich eines Internationalen Symposiums beschrieben worden.

Abb.4 (S. 61) deutet an, daß das Vorherrschen der Pneumokoniose in der britischen keramischen Industrie dramatisch gefallen ist; aber solange man noch mit Materialien arbeitet, die über $20 \%$ Kieselsäure enthalten, ist das Ende der via dolorosa der keramischen Arbeiter noch nicht in Sicht.

\section{BUUE ASEESTOS}

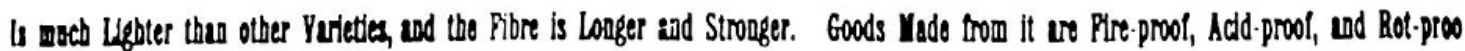

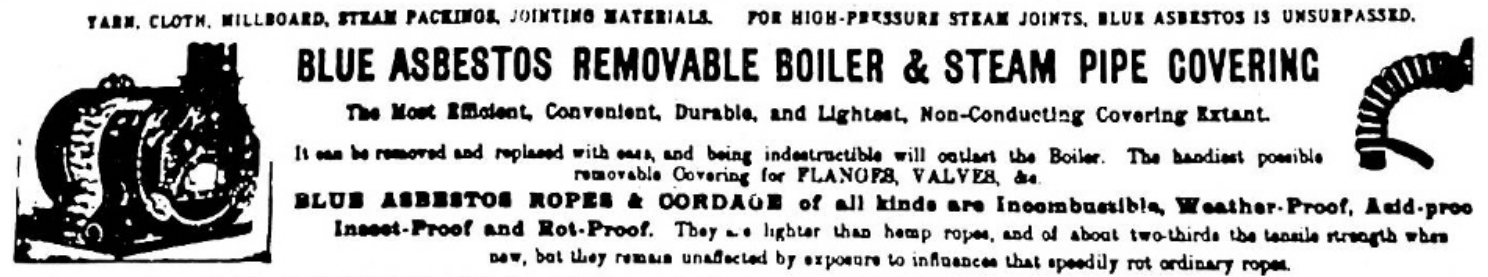

\section{THE CAPE ASBESTOS CO., LTD., 8, MINORIES, LONDON, E.C Mines: Cape Colony. Factories: London, Italy, France.

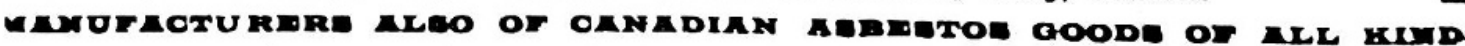

Abb. 2. Inserat für blauen Asbest in der englischen Fachzeitung The Engeneer vom 9. Juli 1897

Wir wenden uns jetzt der Asbestgefahr zu. Obzwar Asbest schon im Altertum für Lampendochte, zur Verstärkung primitiven Geschirres und für Leichentücher verwendet wurde, begann die wirkliche Auswertung der verschiedenen Formen des Asbests erst gegen das Ende des letzten Jahrhunderts. Das Inserat aus einer 
englischen Zeitung auf $\mathrm{Abb} .2$ ist historisch wichtig, da es die weitaus gefährlichste Asbestform, den blauen Asbest (Krozidolit), empfiehlt. Zur Zeit dieser Eulogie betrug der Weltverbrauch aller Asbestarten etwa 500 Tonnen; heute werden über 5 Millionen Tonnen verarbeitet. Die häufigste Form ist der weiße Asbest (Krysotil). Er bestreitet etwa 95\% des Weltverbrauches. Die Fasern des blauen Asbests werden nur in Südafrika und Australien gegraben. Sie liefern nur etwa $4 \%$ der Industrie. Asbest wird für tausend Zwecke benützt, hauptsächlich für feuerfeste Textilien, im Schiffsbau, in der Automobilindustrie und besonders zur Isolierung.

Nach Cooke (1924) wurde der erste Fall von Asbestosis von Murray 1899 in London festgestellt. Es dauerte über dreißig Jahre, bis in England beschränkte Schutzmaßnahmen für gewisse Asbestarbeiter eingeführt wurden, und erst ganz kürzlich wurde die Gesetzgebung auf alle Asbestarbeiter ausgedehnt (Department of Employment and Productivity, 1969). Abgesehen von der Pneumokoniose der Kohlenarbeiter, ist heute in England die Asbestose die häufigste pensionsberechtigte gewerbliche Staubkrankheit.

Im Gegensatz zu den meisten anderen Pneumokoniosen hat der Asbest einen engen und wahrscheinlich kausalen Zusammenhang mit bösartigen Tumoren. Die Häufigkeit des Lungenkrebses bei Asbestarbeitern wurde schon vor dem letzten Weltkrieg von amerikanischen Pathologen beschrieben (Lynch und Smith 1935). Ihre Befunde wurden von Doll 1955 in England einwandfrei bestätigt. Zigarettenrauchen hat sich bei Asbestarbeitern als ein wichtiger co-carcinogener Faktor erwiesen (Berry et al. 1972).

1960 haben Wagner und seine Mitarbeiter in Südafrika auf die relative Häufigkeit von pleuraler und peritonealer Mesotheliomata bei den Einwohnern in der Nähe von Asbestgruben hingewiesen. Nicht lange darauf wurde die Beziehung dieser immer tödlichen Tumoren, die bisher in den Lehrbüchern kleingedruckt waren, mit der Einatmung von Asbeststaub erklärt (Newhouse und Thompson 1965). Seit 1964 sind in Großbritannien mehr als 600 und in Südafrika über 200 Fälle dieser Tumoren gezählt worden, und es besteht kein Zweifel mehr, daß der blaue Asbest (Krozidolit) der Hauptschuldige ist. Die Gefahr beschränkt sich nicht auf die Arbeiter, sondern betrifft auch die Einwohner in der Nähe der Asbestgruben und Fabriken. In England ist jetzt die Einfuhr des blauen Asbests völlig eingestellt worden; aber es ist bedauerlich, daß in den Vereinigten Staaten, in Japan und Deutschland seine Verwendung immer noch im Ansteigen ist (Wagner 1972).

Nun befasse ich mich mit der britischen Kohlenindustrie, da geschichtlich die Fortschritte und Rückschritte in der Verhütung der Kohlenarbeiterpneumo- 
koniose typisch sind. In England grub man schon im 12. Jahrhundert nach Kohle; aber zur Industrie wurde der Kohlenbergbau erst im 16. Jahrhundert, als die englischen Wälder immer weniger Holz lieferten und dieses als Energiequelle sehr teuer wurde (Nef 1932).

Nach der Erfindung der Dampfmaschine stieg der jährliche Verbrauch an Kohle in Großbritannien stark an. Damals beschäftigte die Industrie 320000 Arbeiter, darunter viele Frauen und Kinder. In einem parlamentarischen Bericht von 1842 finden wir den Absatz: «Die Saat tödlicher Krankheiten in den Kohlengruben wird sehr oft im Kindesalter gelegt. Jede Generation dieser Klasse ist schon im Alter von 50 Jahren ausgestorben.»

Die Frage: «Warum sterben die Häuer so früh ?» wurde in wissenschaftlichen Kreisen viel besprochen, aber erst spät beantwortet. Natürlich gab es in den Gruben viele Unfälle, Unterernährung und Überarbeit, Leptospirose und Phthise; jedoch am Ende des 18. Jahrhunderts trat der Begriff «schwarze Lunge» immer mehr in den Vordergrund.

In Frankreich versuchte Laënnec (1826), die «wirkliche Melanose» und die «Matière noire pulmonaire» der Stadtbewohner zu unterscheiden. Er fand auch in den Lungen der Kohlenarbeiter fibröse schwarze Massen, die er mit einem «morceau de savon noir» verglich und die wahrscheinlich die heutige progressive massive Fibrose war. Die meisten französischen Autoren betrachteten die «matière noire» als ein endogenes Krankheitsprodukt, während Laënnec vermutete, daß das Einatmen von Rauch und Ruß seine Ursache war. Die beste Beschreibung der schwarzen Lunge in der Weltliteratur findet man in Emile Zolas « Germinal» (1885).

Die deutschen Pathologen folgten viele Jahre dem Dogma ihres Meisters Rudolf Virchow, der kategorisch die Möglichkeit der Kohlenstaubeinatmung verneinte und die schwarze Lunge mit extravasiertem Hämatin erklärte (Virchow 1847).

Im Gegensatz dazu erklärte Pearson (1813) in der Royal Society in London in einer Abhandlung über die schwarzen Bronchialdrüsen und Lungenflecke, daß die schwarze Lunge auf der Einatmung unsichtbarer Kohlen- und Rußstäubchen beruhe. Ein anderer schottischer Arzt, W.Marshall, glaubte, daß die «spurious melanosis» (unechte Melanose) auf die Einatmung des Kohlenstaubes und nicht auf Lampenruß zurückzuführen sei (1833/34). 1831 berichtete J.Gregory über einen Fall von «sonderbarer schwarzer Infiltration der Lungen», die er als spezifische Kohlenarbeiterkrankheit betrachtete. Aus Schottland stammt auch die erste Beschreibung des sogenannten «perifokalen Emphysems» (Craig 1834), das erst nach weiteren hundert Jahren von Gough (1949), Hepplestone (1951) und anderen als charakteristisches Merkmal der unkomplizierten Kohlenpneumo- 
koniose erkannt wurde. 1837 zerhieb Stratton den gordischen Knoten der verwirrten Nomenklatur und schlug den Namen «Anthrakosis» vor, der bald von den meisten Autoren angenommen wurde.

Ein bedeutsames Jahr war 1867, als Zencker in Bayern eine «rote Lunge» einer Arbeiterin beschrieb, die hohen Konzentrationen von Eisenpigment ausgesetzt gewesen war. Er prägte das Wort «Siderose» und schrieb die prophetischen Worte: «Wir haben jetzt zwei Arten dieser Krankheit kennengelernt, die Anthrakose und die Siderose. Wir können annehmen, daß in der Zukunft ähnliche Staubkrankheiten entdeckt werden. Es wird notwendig sein, alle diese Krankheiten unter einem einzigen Titel zu erfassen, und der Name Pneumokoniosis empfiehlt sich uns. » Einige Jahre später gab endlich Visconti (1870) dem Urvater aller Pneumokoniosen einen wissenschaftlichen Namen: Silikosis.

Die meisten Medizinhistoriker sind sich einig, daß im 19. Jahrhundert das Hauptverdienst in der Erkennung und Klassifizierung der Staublungen dem Engländer E.H. Greenhow zugesprochen werden muß. Greenhow bereiste alle Grubengebiete und Industriezentren; er war ein hervorragender Histologe, der zum ersten Male in diesem Feld polarisiertes Licht anwandte. In seinem Bericht (1862) ließ er keinen Zweifel darüber bestehen, daß die Krankheiten, die im Volk als Töpferfäule, Schleifer-Asthma und Bergsucht bekannt waren und die von den Wissenschaftlern als schwarze Lunge, Phthisis melanotica und interstitielle Lungenentzündung und Fibrose beschrieben wurden, eine und dieselbe Ursache hatten: die Einatmung unsichtbarer industrieller Staubteilchen.

\section{Von der Leugnung zur Anerkennung der Staublunge}

Gegen das Ende des 19. Jahrhunderts verschwand in medizinischen und technologischen Kreisen das Interesse für die schwarze Lunge. Die Ursache für diese Vernachlässigung zur Zeit verstärkter Mechanisierung ist heute noch ein ungelöstes Rätsel. Der Hygieniker Adolf Vogt in Bern behauptete (1887), daß die Quarz- und Eisenlunge und die Anthrakose in ein Kuriositätenkabinett, aber nicht in die ernste wissenschaftliche Arbeitshygiene gehören. 1908 erklärte Oliver: «Die schwarze Lunge der Häuer gibt es kaum mehr», und sein Kollege Shufflebotham wiederholte diese Ansicht in einer Festrede (1914): «Heutzutage kommt die Lungenfibrose der Grubenarbeiter kaum mehr vor.» 1915 krönte dies alles einer der hervorragendsten Staubkrankheitsforscher, Collis, mit den Worten: «Das Asthma der Bergarbeiter ist unbemerkt aus unserer Mitte verschwunden, und Spekulationen über seine Ursache sind heute unfruchtbar.» Der berühmte 
Physiologe und Vorstand des britischen Grubeninstitutes, J.S. Haldane, erklärte, daß die Einatmung von Staub keine Gefahr bedeute (1924).

Dr. C. Fletcher hatte sicherlich recht, als er 1918 schrieb : «Solche Äußerungen prominenter Wissenschaftler waren großenteils verantwortlich dafür, daß die Grubeningenieure zur Zeit der intensiven Mechanisierung in ihren Bemühungen zur Staubunterdrückung nachließen.»

Im dritten Jahrzehnt unseres Jahrhunderts waren Pneumokoniosis und Silikosis noch immer synonym, und das zentrale Dogma blieb auf der internationalen Silikosekonferenz in Johannesburg unerschüttert (Gardener et al. 1930): «Ohne freie Kieselsäure keine Silikose.»

Als in England 1919 die Silikose endlich als pensionsberechtigte Berufskrankheit anerkannt wurde, mußten die Antragsteller auf eigene Kosten beweisen, daß sie Staub mit wenigstens $80 \%$ Kieselsäuregehalt ausgesetzt gewesen waren. Dieser Vorbehalt disqualifizierte natürlich die Mehrheit der Kohlen- und Porzellanarbeiter und beinahe alle Angestellten in den Eisen- und Stahlgießereien.

Während die Ursache und Bedeutung der Kohlenarbeiterpneumokoniose noch eifrig bestritten wurde, hatte sich die Röntgentechnologie so sehr verbessert, daß man mit guten Apparaten nicht nur große Schatten und Kavernen, sondern auch kleine Herde erkennen und abbilden konnte. Ein Schweizer mit dem angemessenen Namen Staub-Oetiker verwendete die Lungenröntgenologie 1916 erfolgreich in Serienuntersuchungen von Metallschleifern. Weitere Pioniere dieses Verfahrens finden wir in Australien (Gandevia 1973), wo Nelson bereits 1926 röntgenologische, klinische und physiologische Reihenuntersuchungen an Bergarbeitern Westaustraliens durchführte. Nelson scheint auch der erste Autor zu sein, der feststellte, daß nicht das Ausmaß der röntgenologischen Veränderungen, sondern die physiologische Dysfunktion der Lungen als Grundlage einer rationellen Pensionspolitik zu betrachten ist. In einem anderen Dominion des ehemaligen Britischen Reiches lieferte das südafrikanische Grubenbureau nach der Entdeckung der reichen Goldflöze wichtige Beiträge zur Diagnose und Verhütung der Silikose, die im einzelnen von Meiklejohn (1952) und Orenstein (1957) beschrieben wurden.

In den zwanziger Jahren tauchte das Gespenst der Kohlenarbeiterpneumokoniose wieder in England auf, und zwar diesmal in Wales. Die Verbitterung der arbeitsuntauglich gewordenen, aber pensionsdisqualifizierten Häuer stieg von Jahr zu Jahr, besonders während der großen wirtschaftlichen Weltkrise. 1930 behaupteten Cummins und Sladen, daß die chronische Kohlenarbeiterlunge eine Krankheit sui generis sei. Sutherland und Bryson (1926) fanden bei den Röntgenaufnahmen der Geschirrarbeiter Veränderungen, die man nicht als klassische 
Silikose betrachten kann, und später fanden McLoughlin und seine Mitarbeiter bei Eisen- und Stahlgießereiangestellten ähnliche Veränderungen, denen sie den Namen «Mischstaubpneumokoniose» gaben (McLoughlin et al. 1950).

Während des letzten Krieges entschloß sich endlich die britische Regierung, die chronischen Brustkrankheiten der Grubenarbeiter in Wales gründlich untersuchen zu lassen. Das Ergebnis, der Bericht von D'Arcy-Hart und Aslett, brachte entscheidende neue Erkenntnisse über die Kohlenarbeiterlunge:

1. Die röntgenologischen Veränderungen in den Lungen der Kohlenarbeiter unterscheiden sich wesentlich von denen der klassischen Silikose.

2. Das erste röntgenologische Zeichen der Kohlenarbeiterpneumokoniose ist die sogenannte Retikulation, die, wie Gough (1949) und Hepplestone (1951) zeigten, aus kleinen Kohlenknoten mit umgebenden «fokalen Emphysemen » besteht.

3. Die Kohlenarbeiterpneumokoniose findet man nicht nur in Anthrazitgruben, sondern auch in bituminösen Revieren.

Auf Grund dieser Befunde beantragte das offizielle Komitee für Industriekrankheiten die Definition der Pneumokoniose für Pensionszwecke folgendermaßen: «Die Pneumokoniose ist eine Lungenfibrose, verursacht durch Silikat- und Asbeststaub und andere Staubarten, und sie schließt die sogenannte Retikulation ein.»

Abb. 3. Fälle von Pneumokoniose bei Kohlenarbeitern, die deswegen in Großbritannien 1948-1972 pensioniert wurden

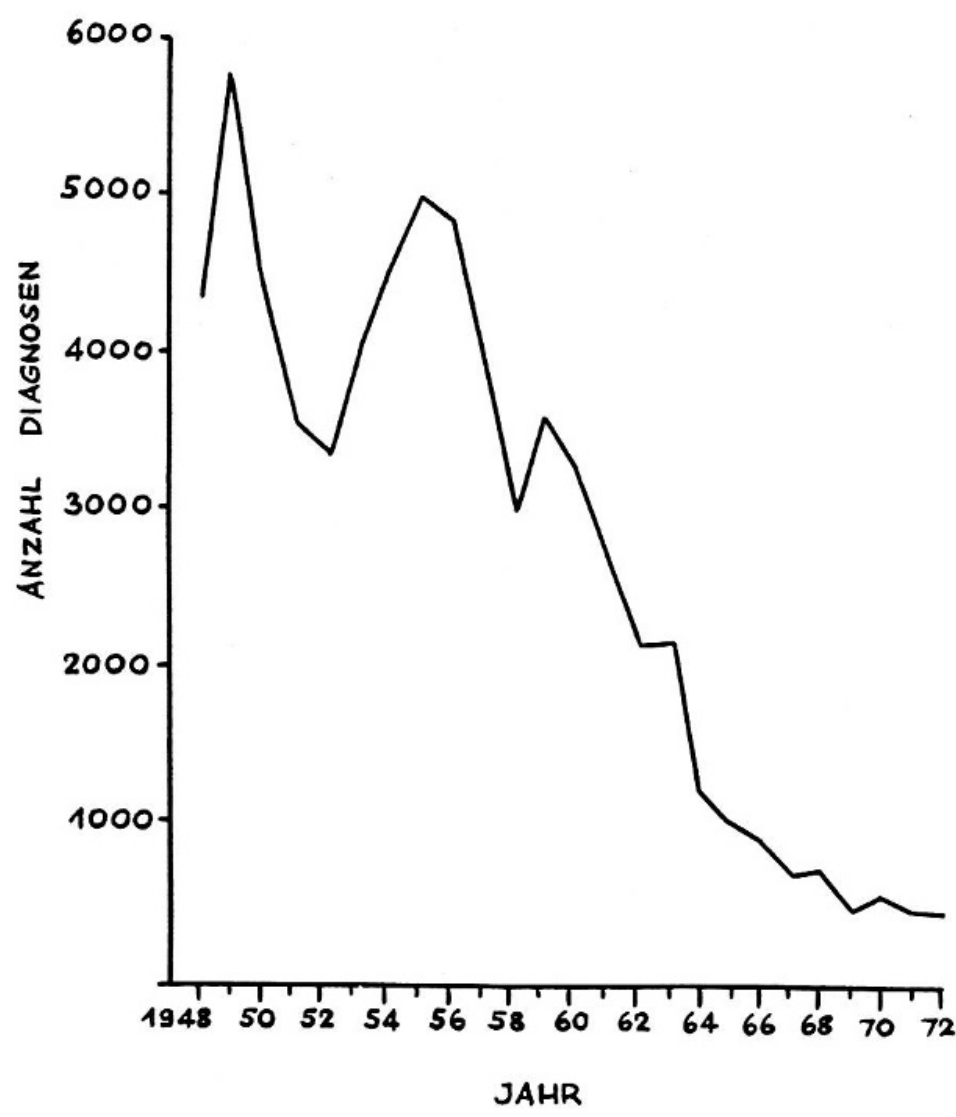


Wie auf Abb. 3 zu sehen ist, führte diese Erweiterung der Definition anfangs zu einer Lawine von Pensionsanträgen, die aber bis zum Jahre 1967 ständig fielen. Trotz der Schließung vieler Kohlengruben in Großbritannien ist seit jenem Jahre die Anzahl der jährlich entschädigten Grubenarbeiter kaum mehr gefallen.

Schließlich führte die Untersuchung von D'Arcy-Hart und Aslett zur Gründung eines permanenten Pneumokoniosis-Institutes in Wales, das im Laufe der Jahre das Mekka der Pneumokoniose-Forscher und -Epidemiologen wurde. Zur selben Zeit wurde die gesamte Kohlenindustrie verstaatlicht, was den Aufbau eines wirksamen Gesundheitsdienstes in den Kohlengebieten sehr erleichterte. Insbesondere die regelmäßigen Reihenuntersuchungen der Grubenarbeiter haben sehr viel zu unserer Kenntnis des Vorherrschens der Pneumokoniose beigetragen. Ähnliche Institute wurden auch in vielen anderen Staaten, einschließlich der Schweiz, aufgebaut.

Die wichtigsten Erfolge des «Pneumoconiosis Unit» in Wales, des Gesundheitsamtes des National Coalboard und anderer Untersuchungsstellen in mehreren Ländern scheinen die folgenden zu sein:

1. Die Planung breiter vielseitiger Felduntersuchungen, die nicht nur die Grubenarbeiter, sondern auch alle Einwohner gewisser Gebiete umfassen.

2. Die Verknüpfung der Arbeitsbedingungen, insbesondere quantitativer und qualitativer Staubmessungen, mit biologischen Reaktionen, die von der Röntgenologie, Lungenphysiologie und Immunologie erfaßt werden.

3. Die Einführung einer zweckmäßigen und einfachen Terminologie der Röntgenerscheinungen, die nationale und internationale Vergleiche erlaubt.

4. Die Entwicklung der Lungenphysiologie auf der Grundlage einfacher und verläßlicher Apparate.

5. Die vielfachen Vergleichsmöglichkeiten lassen die Beobachtungsfehler bei der radiologischen, physiologischen und klinischen Untersuchung eingrenzen, was zu zuverlässigeren statistischen Analysen geführt hat.

In Industrien mit Staubgefahr sind international weitgehende technologische Schutzmaßnahmen eingeführt worden. Viele dieser Fortschritte beruhen auf ärztlichem Rat im Gebiet der Diagnose. Die Therapie ist weniger vorangekommen. Im Augenblick können wir nur die Komplikationen der Staubkrankheiten, wie Tuberkulose, andere respiratorische Infektionen und manchmal einen kardiorespiratorischen Zusammenbruch, erfolgreich behandeln. Ob jemals eine medizinische Behandlung der unkomplizierten Pneumokoniosen möglich sein wird, ist zweifelhaft. Der Nachdruck muß daher auf der Vorbeugung liegen. 
Abb.4. Pneumokoniose in Großbritannien 1953-1972

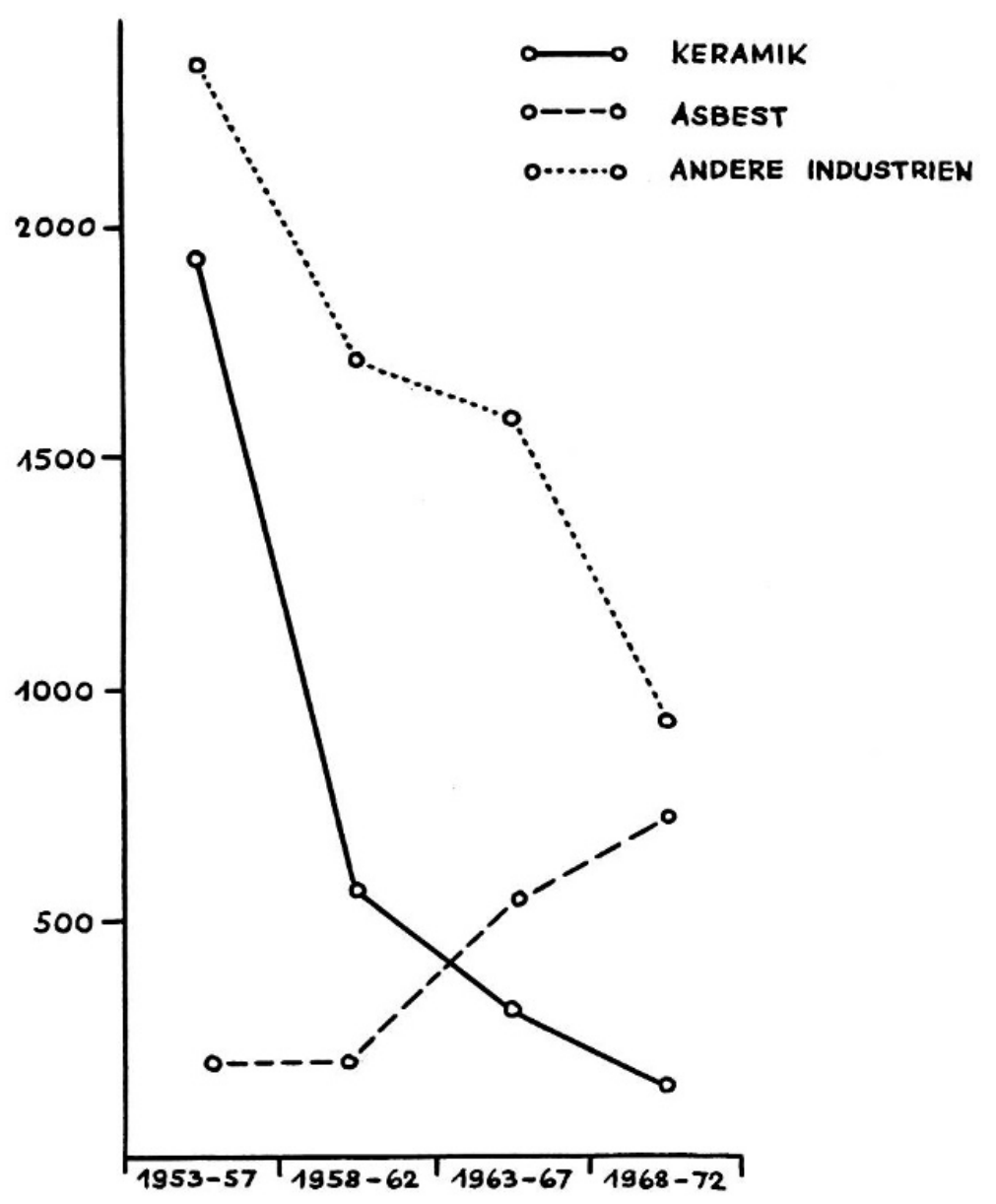

Die Abb. 4 zeigt, wie sehr sich die Verhältnisse in Großbritannien außer bei der Asbestose verbessert haben. Jedoch die einzige Lösung des Problems wäre die vollkommene Staubverhütung und die Verwendung harmloser statt fibrogenöser Stoffe. Beides ist noch ein Traum.

Ferner müssen wir zugeben, daß seit Agricola und Paracelsus zwar viele Hypothesen über die Naturgeschichte der Mineral-Pneumokoniosen vorgeschlagen worden sind, daß wir aber immer noch nicht wissen, warum ein Mann nach vierzig Arbeitsjahren ein respiratorischer Krüppel wird oder an einer fortgeschrittenen Pneumokoniose stirbt, während sein Nachbar, der mit ihm ebensolange unter denselben Bedingungen gearbeitet hat, mit einer leichten und nicht beschwerlichen Pneumokoniose davonkommt. In den letzten Jahren hat sich die Wissenschaft viel mit der Immunologie und sogar mit genetischen Aspekten der Staubkrankheiten beschäftigt; einwandfreie Ergebnisse liegen jedoch noch nicht vor.

Ignoramus, ignorabimus? Wir können das heute noch nicht beantworten. In der Zwischenzeit muß das Hauptziel die Vorbeugung bleiben. 


\section{Literaturangaben}

Agricola G. (1556), De re metallica libri XII, Basel.

Arlidge J.T. (1892), The Hygiene, Diseases and Mortality of Occupations, London.

Berry G., Newhouse M.L. \& Turok M. (1972), Combined Effect of Asbestos Exposure and Smoking on Mortality of Lung Cancer.

Collis E. (1915), Industrial Pneumoconiosis with special reference to Dust Phthisis. London, H.M.S.O.

Cook W. E. (1924), Fibrosis of the lungs due to Asbestos Dust. Brit. Med. J. 2, 147.

Craig W. (1834), Observations on spurious melanosis. Edinburgh Med.Surg. J.42, 330.

Cummins S. L. \& Sladden A. F. (1930), Investigations into the Anthracotic Lungs of Coalminers in S. Wales. J.Path. Bac. 33, 1095.

Dept. Employment and Productivity (1969), Asbestos Regulations. London H.M.S.O.

Diemerbroeck Y. van (1679), Anatome corporis humani, Leyden.

Diodorus Siculus (1.Jh.v.Chr.): nach Rosen, The History of Miners Diseases, New York 1943.

Doll R. (1955), Mortality from Lung Cancer in Asbestos Workers. Brit. J.Indust. Med.12, 81 .

Ellenbogen U. (1524), Von den gifftigen, besen Tempffen und Reuchen, Augsburg.

Farr W. (1877), Thirty Eighth Annual Report of the Registrar General for 1875, London H.M.S.O.

Fletcher C. H. (1948), Pneumoconiosis of Coalminers. Brit. Med. J.1, 1015 \& 1065.

Gandevia B. (1973), The Australian Contribution to the History of Pneumoconiosis. Med. Hist. 17, 368.

Gardener L. W., Middleton E. \& Orenstein A.J. (1930), Report on the Medical Aspects of Silicosis. Resolution of the International Conference on Silicosis, Johannesburg. Supplement S.3-8.

Gough J. (1949), The Pathology of Pneumoconiosis. Postgrad. Med. J.25, 611.

Greenhow E.H. (1862), Report of the Medical Officer of the Privy Council, Appendix VI (nach Meiklejohn 1951).

Gregory J.C. (1831), Case of peculiar black infiltration of the lungs, resembling melanosis. Edinb. Med.J.36, 389.

Haldane J.S. (1924), Historical Review of Coalmining. Kapitel 18. London.

Hansard (1842), House of Lords, June 24th.

Hartin F. H. \& Hesse W. (1879), Der Lungenkrebs, die Bergkrankheit der Schneeberger Gruben. Vierteljahrsschr. Gerichtl. Med.30, 296; 31, 102 \& 313.

Hepplestone A.G.H. (1951), Coalworkers Pneumoconiosis. A.M. A. Arch. Ind. Hyg. Occup. Med. 4, 270.

Laënnec R.T.H. (1826), Traité de l'auscultation médiate, $2^{\mathrm{e}}$ édition, Paris.

Löwy J. (1929), Über die Joachimsthaler Bergkrankheit. Med. Klin.25, 141.

Lucretius Carus, De rerum natura, Liber VI. 
Lynch K.M. \& Smith W.A. (1935), Pulmonary Asbestosis. Am. J. Cancer 24, 56. Marshall W. (1833/34), Cases of spurious melanosis of the lungs or phthisis melanotica. The Lancet, 271.

McLoughlin A.I.G., Cheeseman C.A., Garrod J., Gloyne S.R., Goodall E.C., Harding J.E., Jupe A.H., Lawrie W.P., Perry K.A., Sutherland C.L. \& Woods H. (1950), Industrial Diseases of Iron and Steel Workers. London H.M.S.O.

Meiklejohn A. (1951), History of Lung diseases in Coalminers in Great Britain. Part I. Brit. J. Indust. Med.8, 127.

Meiklejohn A. (1963), The successful prevention of Silicosis among China Biscuit workers in North Staffordshire. Brit. J.Indust. Med.14, 211.

Meiklejohn A. (1969), The History of Occupational Respiratory Disease in the North Staffs. Pottery Industry. In: Health Conditions in the Ceramic Industry. Ed. C.N.Davies, Pergamon Press, Oxford.

Meiklejohn A. \& Posner E. (1957), The Effect of the Use of Calcined Alumina in China Biscuit Placing on the Health of the Workman. Brit. J.Indust. Med. 14, 229.

Nelson W.T. (1926), Report on the investigation of the Pulmonary Condition of Mine Employees in Western Australia. Service Public.V.Div.Indust. Health, Canberra.

Newhouse M.L. \& Thompson H. (1965), Mesothelioma of the Pleura and Peritoneum following exposure to Asbestos in London area. Brit. J.Indust. Med.22, 161.

Nef J. U. (1932), The Rise of the British Coal Industry, London.

Oliver T. (1908), Diseases of Occupation, London.

Palmer P.E.S. \& Daynes G. (1967), Transkei Silikosis. S. Afr. Med.J.41, 1182.

Pansa M. (1614), Consilium peripneumoniacum. Das ist ein guter Rat usw., Annaberg.

Paracelsus Theophrastus von Hohenheim (1567), Von der Bergsucht oder Bergkranckheiten, Dillingen. - In Sudhoffs Paracelsus-Gesamtausgabe: Band 9, München-Planegg 1925, S.461-562 (Rezept S.485).

Parliamentary Papers (1842), XV-XXII, London H.M.S.O.

Pearson G. (1813), On the colouring matter of the black bronchial glands and of the black spots in the lungs. Phil. Trans. Royal Soc. London 103, 159.

Plinius Secundus, Naturalis historia, libri 33-37. Wiederabdruck bei Detlefsen, Berlin 1873.

Posner E. \& Kennedy M.C.S. (1967), A further study of China Biscuit Placers in Stoke-on-Trent. Brit. J. Indust. Med.24, 133.

Ramazzini B. (1700), De morbis artificum, Modena.

Rosen G. (1943), The History of Miners Diseases, New York.

Shufflebotham S. (1914), The Hygienic Aspects of the Coalmining Industry in the United Kingdom. Brit. Med. J. 1, 558.

Silicus Italicus (1.Jh.v. Chr.): nach Rosen 1943.

Staub-Oetiker (1916), Die Pneumokoniose der Metallschleifer. Deutsch. Arch.f. klin. Med. 61, 469. 
Stratton T. (1837), Case of Anthracosis or Black Infiltration of the Lung. Edinburgh Med. and Surg. J.49, 490.

Schiller F. (1974), persönliche Mitteilung.

Sutherland C.L. \& Bryson S. (1926), Report on the Incidence of Silicosis in the Pottery Industry. London H.M.S.O.

Virchow R. (1847), Die pathologischen Pigmente. Arch. Path. 1, 434, 461.

Visconti L. (1870), Protocollo generale delle necropsie seguite nell'Istituto Anatomo-Patologico dell'Ospedale Maggiore.

Vogt A. (1887), Die allgemeine Sterblichkeit und Sterblichkeit an Lungenschwindsucht in den Berufsarten. Zeitschr.f. schweiz. Statistik 23, 249.

Wagner J.C. (1972), Current Opinions on the Asbestos Problem. Ann. Occ. Hyg. 15,61 .

Wagner J.C., Sleggs C.H. \& Marchand P. (1960), Diffuse Pleural mesothelioma and Asbestos exposure in the Cape Province. Brit.J. Indust. Med.17, 260.

Zencker A. (1867), Über Staubinhalationskrankheiten der Lungen. Deutsch. Arch. Klin. Med.2, 116.

Zola E. (1885), Germinal, Paris.

\section{Summary}

Silicosis must be one of the eldest diseases. In ancient times the labour in mines was like a sentence of death. George Agricola wrote in 1556 that the dust enters the respiratory tract, causes shortness of breath, and the miners die of pulmonary diseases. Ysbrand van Diemerbroeck described in 1672 that his knife gnashed, when he draw it through a stone-mason's lung. The industrial revolution increased the danger in the china and asbestos industry and in coal-mining. Astbury's invention of finely grinded flints was good for the ceramics of Stoke-on-Trent, but bad for the health of the potters. A physician, John T. Arlidge, published a clear treatise thereon in 1892. - Only in newer times one recognized the endangering of the asbestos-workers. - The steam-engines required much coal. Many coalminers died early. Pathologists saw that their lungs were black. Pearson explained in 1813 , that the cause was the inhalation of invisible coal-particles. Greenhow confirmed this opinion in 1862. Nevertheless, later scientists maintained that the inhalation of dust was not dangerous, so that the engineers neglected the fight against it. Only slowly one realized that silicosis and pneumoconiosis are occupational diseases and give the right to a pension. Because no therapy exists, prevention is important.

Dr.med. Erich Posner

Central Outpatients Department

Stoke-on-Trent

England 